\title{
Control and Management of Energy in a PV System Equipped with Batteries Storage
}

\author{
Kamal Hirech , Mustapha Melhaoui, Khalil Kassmi* and Rachid Malek \\ * Laboratory LETSER, Dept of Physics, Faculty of Science, University Mohamed I, Oujda, Morocco. \\ E-mail: khkassmi@yahoo.fr
}

\begin{abstract}
In this paper the researchers present a work concerning the conception, implementation and testing of a photovoltaic system that is equipped with a new concept of control and management of energy in a PV system with battery storage. The objective is to exploit the maximum of power using Hill climbing improved algorithm that considers optimal electrical characteristics of PV panels regardless of the system perturbation, to manage the energy between blocs of PV system in order to control the charge/discharge process and inject the energy surplus into the grid and also to estimate the state of charge with precision. Moreover, the system guarantees the acquisition and presentation of results on computer, supervision and so on. The results obtained show the robustness of the PV system, good control and protection of batteries under the maximum of energy provided by the PV panels. The state of charge estimation is evaluated by using measured parameters in real time; it shows an improvement of around 5\% compared to the conventional technique.
\end{abstract}

Keywords - regulator; photovoltaic system; charge/discharge; Battery; SoC, MPPT; Converter.

\section{INTRODUCTION}

Nowadays, there are many works about photovoltaic systems (PV) that operate the energy in order to inject it into the grid [1] or in the stand-alone installations. [2]

The autonomous systems or those connected to the grid [1-3] are equipped with batteries in order to satisfy the needs of the local demand of energy [4] or facilitate coupling and solve the problems of adaptation between production and demand [3-4]. The major challenges for these structures are the protection, stability and storage of energy when the batteries are fully charged. However, those batteries suffer from various types of degradation due to their bad use, a bad control of charging/discharging, and the impact of temperature.

So, all optimization of the life-time of the battery requires a system to control and manage its functioning.

In This paper, the researchers study a structure of a photovoltaic system equipped with a new concept to control and manage the energy supplied by panels in the charge/discharge process with a good precision of the state of charge estimation (SoC), this is by a regulation system based on: Firstly the hash of current supplied by panels via a PWM signal controlling the switch Buck or Boost DC/DC converter to maximize the power to the output and secondly, the hash of current supplied to the output of the converter to charge the batteries.

A part of energy produced and unabsorbed by the batteries during the charging is injected into the grid with the minimum of energy loss. All functioning tasks of this system, which are under optimal conditions by maximization of the power transferred to the converter output, increase the rapidity of charging and improve efficiencies by an improved Hill climbing algorithm of MPPT, taking into consideration the optimal electrical characteristics of panels. The state of charge SoC determination is achieved by the combination of two methods to correct estimation errors and to increase precision. The initialization of SoC is fixed by the method of open circuit voltage (OCV) [5] when the batteries are at rest: a first recalibration of the SoC is applied. The quantity of charge absorbed or delivered by the batteries is calculated by the Coulomb Counting method in real time [6] and a second recalibration of the SoC is applied when the batteries reach full charge conditions. The proposed method is characterized by being simple and easy to implement and does not require the use of additional hardware. 
The various measurements and parameters will be displayed on an LCD and transferred through a serial connection to a computer [7].

\section{FUNCTIONING OF THE PV SYSTEM}

\section{A. Architectural PV system}

The synoptic diagram of the PV system designed and tested (Fig. 1) consists of various:

- $\quad$ Two PV panels mounted in parallel [7].

- Two lead acid solar batteries in series.

- DC or AC load through a DC/AC converter $(\operatorname{lamp}, \ldots)$

- $A D C / D C$ Boost (or Buck) converter to adapt the $\mathrm{PV}$ panels to the batteries [7].

- The management and supervision system (MSS), which is based on the use of a microcontroller to perform all the tasks of: optimization of the system functioning by the MPPT control, regulation of charge/discharge process, control of the energy transfer between various blocs, acquisition and display of different quantities (voltages, currents, powers, SoC, performance, illumination, and temperature) on the screen of a PC or LCD and communication to the computer by a serial link [7].

- Block of energy flow controller, formed by three power circuit (circuit 1 to 3 ) presented in Fig .1.

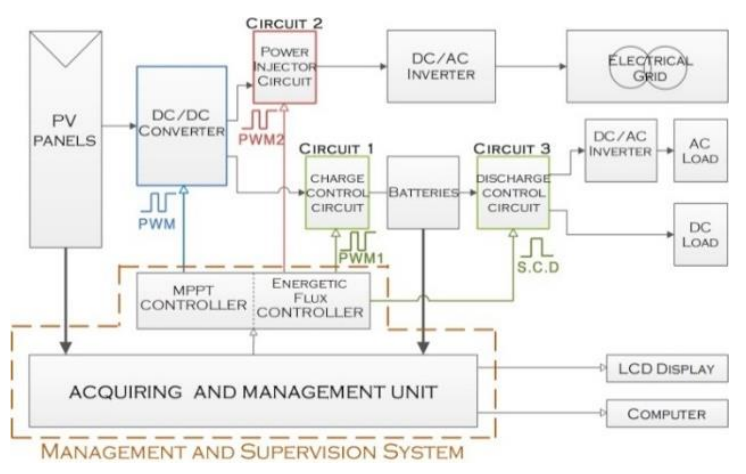

Fig .1. Synoptic diagram of the PV system designed.

\section{B. The MPPT control}

The MPPT control, which is characterized by its robustness and precision [8], generates a PWM signal of a frequency of $10 \mathrm{kHz}$ with variable duty cycle $\alpha$. Its main role is to converge instantly the functioning point of the PV panels to the MPP, regardless of weather or load variations. The principle is to take into account the performance of the PV panels. The improvement provided is to search the PPM in an optimal voltage range which is previously determined by the user depending on panels used and weather conditions, in our case between $12 \mathrm{~V}$ and $16 \mathrm{~V}$. This algorithm is based on the determination of the system evolution from the sign of $\mathrm{dPpv} / \mathrm{dt}$ generated by the panels that forces the system to search MPP between (Vmin, Vmax) by executing the algorithm in Fig. 4. This is by a flow test of the timer TM and the verification of the state of the signal Vstate which allows, at the end of the algorithm, to increment the duty cycle if Vstate $=1$, or to decrement it if Vstate $=0$.

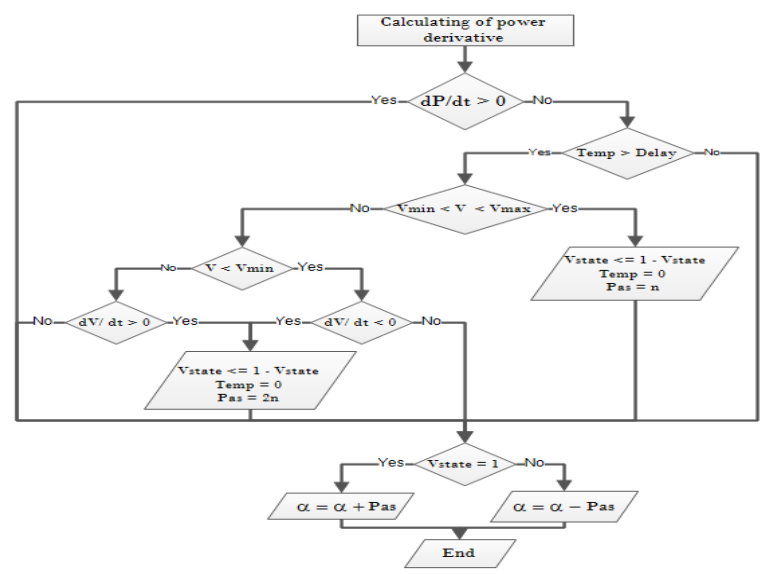

Fig .2. The MPPT algorithm used in this work [8].

\section{Regulation of charging/ discharging process}

The method proposed in this work for the control of energy provided by the PV system is carried out by hashing current supplied to the output of the converter by PWM signals. This energy is used to charge the batteries following three phases described by the algorithm in Fig. 3 and the surplus is injected into the grid. In the case at hand, the charge process is done by using the technical characteristics of the batteries shown in Table 1.

The discharging control of batteries is based on the situation of the installation using the algorithm of Fig. 4. This process is activated/deactivated on the basis of batteries estimation of state of charge SoC:

- If the SOC reaches the limit value SoCmin.D, the process of discharge will stop,

- If the SOC is higher than SoCmin.R, the discharge 
process will be activated,

- If SoCmin.D is $<$ SoC $<$ SoCmin.R, the situation of the installation will determine the charge/discharge process. If the discharge takes place following the normal cycle $(\mathrm{dSoC} / \mathrm{dt} \leq 0)$, the batteries will discharge until SoC=SoCmin.D (Table 1). If a new charge cycle takes place (dSoC/dt $>0)$, the discharge will be blocked until the $\mathrm{SoC}$ reaches SoCmin.R.

Table 1. ELECTRICAL CHARACTERISTICS AND VALUES CHARGE/DISCHARGE PARAMETERS OF BATTERIES AT $25^{\circ} \mathrm{C}$.

\begin{tabular}{|l|l|}
\hline Characteristics and conditions of charge & $\begin{array}{l}\text { Batteries of } \\
\mathbf{1 2} \text { cells }\end{array}$ \\
\hline Nominal voltage & $24 \mathrm{~V}$ \\
\hline Nominal capacity & $110 \mathrm{Ah}$ \\
\hline Voltage of regulation $\boldsymbol{V}_{\boldsymbol{R}}$ & $28.4 \mathrm{~V}$ \\
\hline Voltage of floating $\boldsymbol{V}_{\boldsymbol{F L T}}$ & $27,3 \mathrm{~V}$ \\
\hline Current of over charge terminate Ioct & $1 \mathrm{~A}$ \\
\hline Limit Voltage of discharge LVD & $22.8 \mathrm{~V}$ \\
\hline State of charge. minimum to disconnect SoCmin.D & $40 \%$ \\
\hline State of charge. minimum to reconnect SoCmin. $\boldsymbol{R}$ & $45 \%$ \\
\hline
\end{tabular}

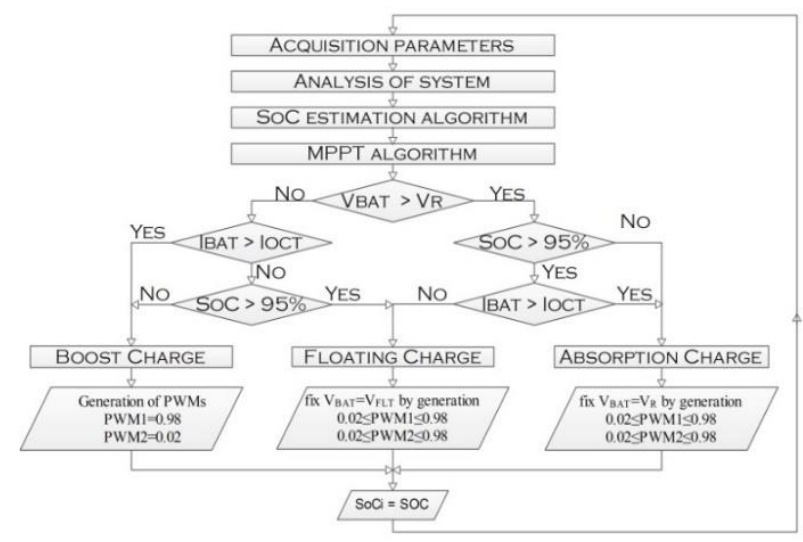

Fig .3. Control algorithm of the batteries charge.

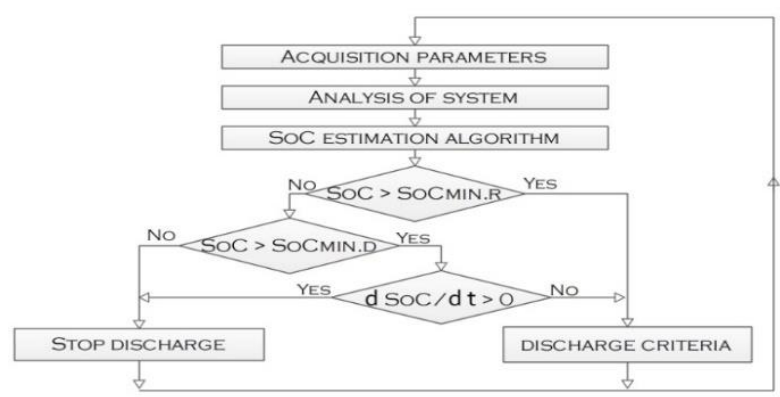

Fig .4. Control algorithm of the batteries discharge.

\section{State of charge estimation}

For a good energy management of batteries, the system must be equipped with an estimation model of the state of charge in order to know the real state of the battery during its use. In this system, this is guaranteed by a model that incorporates the use of two methods for a good estimate. For a battery of a nominal capacity Qo, the initial state of charge SoCi is estimated by the OCV method [9].

The quantity of charge absorbed or delivered is calculated by an algorithm that integrates the current during the charge $\left(I_{B A T}\right)$ and discharge (Idech) as a function of time and taking into consideration the faradaic efficiency ( $\eta)$. A recalibration of $S O C$ is activated when the batteries reach the condition of the final charge as they are fully charged to minimize errors of integration and the effects of temperature. During the charge/discharge, the expression of the SoC is [10]:

$\left.\mathrm{SoC}(\mathrm{t})=\mathrm{SoCi}+[100 \% / \mathrm{Qo}] \times \mid \int(\eta \times \operatorname{Ibat}(t)-\operatorname{Idech}(t)) d t\right]$

The initial state of charge is determined by OCV method following the empirical equation (Eq.(3)) [11]:

$\operatorname{SoCi}(\mathrm{T})=\operatorname{SoC}\left(25^{\circ} \mathrm{C}\right) \times(1+0.003 \times(T-25))$

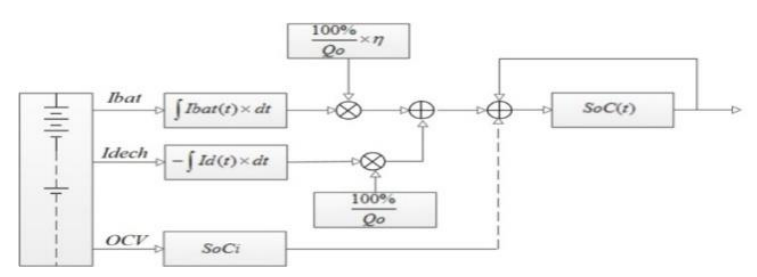

Fig .5 The proposed method to estimate the state of charge SOC.

\section{EXPERIMENTAL RESULTS}

\section{A. Experimental procedure}

Fig. 5 presents the fully automated bench of electrical measurement and the PV system designed and tested when this system is equipped by:

- Two monocrystalline PV panels mounted in parallel. Each one provides, under optimum conditions, a power of $65 \mathrm{~W}$ at the voltage $14.2 \mathrm{~V}$ and a current of $4.4 \mathrm{~A}$. [7]. 
- Two lead acid batteries mounted in series. Each one has a nominal voltage and capacity of $12 \mathrm{~V}$ and $110 \mathrm{Ah}$.

- A DC / DC boost converter dimensioned to operate at a frequency of $10 \mathrm{kHz}$, a power of about $200 \mathrm{~W}$ and a current ranging between $1 \mathrm{~A}$ and $10 \mathrm{~A}[7]$.

- A management and supervision system (MSS) (Fig. 6), which allows management of the acquired data to control the communication between the different blocks of the PV system. This ensures a good functioning of the MPPT control, regulation, control of the energy flow and estimation of the SoC. In addition, it displays all electrical parameters and control switches. A serial link is used to transfer data and store them in a computer.

- The power circuits (circuits 1 to 3 ) controlled by PWM signals whose form ensures the good functioning of energy transfer.

- A weather station equipped with a pyranometer and temperature sensor.

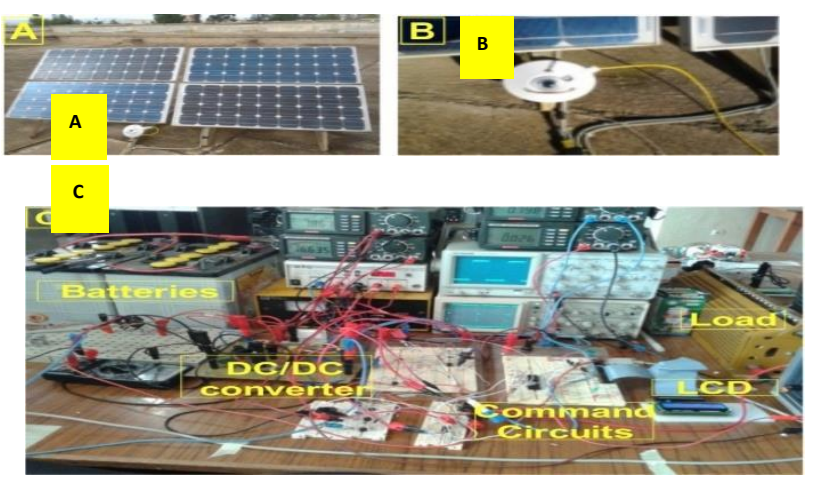

Fig .5 The photovoltaic system designed and digital bench of measurement installed at the Laboratory: $(A)$ : photovoltaic panels.

(B): pyranomete. (C): designed system (batteries, converter, measurement instrument, LCD...).
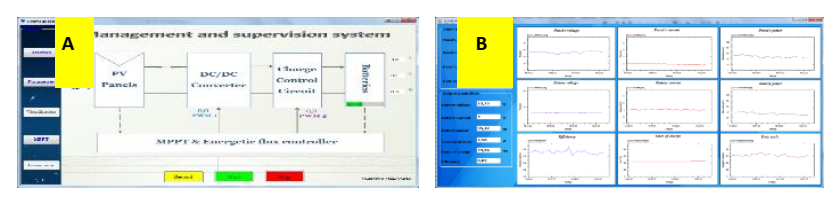

Fig .6 Management and supervision system: (A): global interface. (B): electrical quantities visualization.

\section{B. Energy flow management in the PV system}

1. Batteries regulation charge
During 8 days (D1 to D8), the batteries charging system conceived is analysed (Fig. 5) where the irradiance reaches $800 \mathrm{~W} / \mathrm{m} 2$ (Fig. 7). At first, When the system starts functioning, the acquisition interface performs measurements of batteries voltage under the conditions of open circuit (OCV $=24.28 \mathrm{~V})$, and then calculates the $\mathrm{SoCi}$. The interface displays a $\mathrm{SoCi}$ $=42 \%$. Fig. 8 and Fig. 9 present different results obtained that show:

- During Boost phase (0h to 38h) (Fig. 8): the batteries are charged with a current IBAT that reaches $2 \mathrm{~A}$ towards midday. The voltage VBAT and SoC increase until the threshold limit VBAT = VR. The SoC shows that more than $90 \%$ of the battery capacity is recovered during this phase (Fig. 9). In the same figure of the SoC, the values obtained by the two methods, that was proposed in this work (Eq.(1)) and OCV method [5] are traced. This latter (the OCV method) requires a very long period of rest. This reflects the estimation errors observed. However, the proposed method does not require a rest period except in the calculation of the SoCi. So a difference of the order of $5 \%$ appears between the two methods. The SoC values estimated by the proposed method coincide perfectly with those concluded from the batteries when these last enter in a long period of rest. Improvement of obtaining SoC by our method as compared to the conventional method can be estimated at $5 \%$.

- During the absorption phase (38h-50h) (Fig. 8): The batteries are charged by current pulses IBAT in order to fix VBAT around $28.4 \mathrm{~V}$ to complete the charging of batteries up to SoC $=95 \%$ (without reaching overcharge). At this phase (Fig. 9), the SoC increases slowly due to the progressive decrease of the charge current absorbed by the batteries. The end of this phase is determined by a SoC of over $95 \%$ and a current above IOCT $=0.8 \mathrm{~A}$. During this phase, the surplus of energy is injected into the grid.

- During the floating phase (50h to $55 \mathrm{~h}$ ) (Fig. 8): the batteries are fully charged (Fig. 9). To compensate self-discharging, the system provides the charge through current pulses of low value $(0.3 \mathrm{~A})$ around the float voltage $\mathrm{VFLT}=27.3 \mathrm{~V}$ (away from the gassing range). So, the totality of the energy is injected into the grid (the batteries absorb a low quantity of energy). 


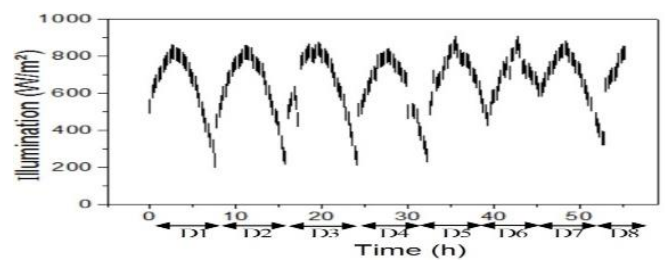

Fig .7 The typical illumination variation during the experimentation days.

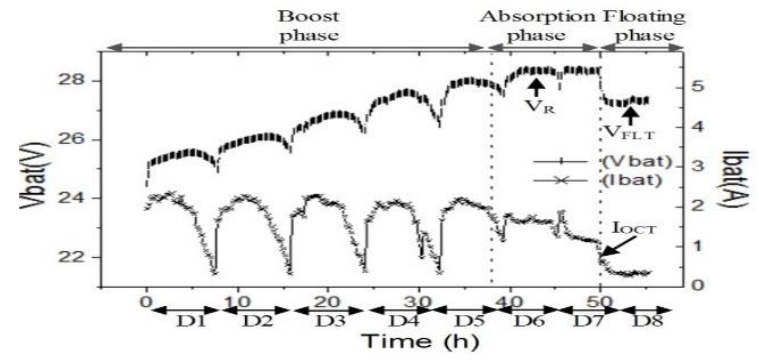

Fig .8 Variation of the charging VBAT and IBAT as a function of time.

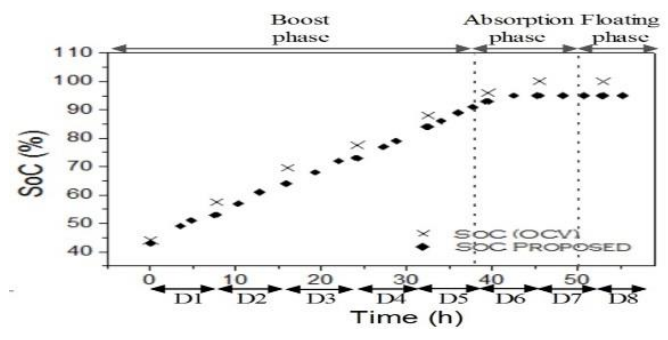

Fig .9 The variation of the state of charge SoC as a function of time.

\section{Management of the batteries energy}

To show the good functioning during the different control modes: charging cycle, discharging cycle and charging/discharging cycle. The PV system is experienced in order to manage the transfer of energy between panels and batteries and also between batteries and loads. The typical results are shown in Fig. 10. In this case, the charging current IBAT is approximately $4.5 \mathrm{~A}$ and the initial battery voltage is VBAT $=12.27 \mathrm{~V}$. While this installation is tested, the management and supervision system (MSS) performs the following operations:

- The initial state of charge estimation $\mathrm{SoCi}=57 \%$.

- During the charge cycle (between 0 and $3 h$ ), when the charge current is IBAT=4.5A and discharge current Idech=0A (Fig.10B), an increase of VBAT and SoC is observed (Fig. 10A). While the SoC reaches $67 \%$.
- During the discharge cycle (between 3 and 8h), when the current Idech=6.5A and IBAT=0A (Fig. 10B) a decrease of VBAT and SoC is observed (Fig. 10A) and when SoCmin.D reached SoC = $40 \%$, the system stops the discharge to protect the batteries (ldech $=0 A)$.

- During the charge/discharge cycle (between 8 and $11 \mathrm{~h}$ ), as long as the SoC is lower than a threshold of reconnection SoCmin. $R=45 \%$ (Fig. $10 \mathrm{~A})$, the discharge is blocked. Above this threshold and depending on the demand, a discharge can take place. When the discharge current is low (Idech=1A) between $9.5 \mathrm{~h}$ and $10 \mathrm{~h}$ (Fig. 10B), VBAT and SoC increase slowly since some of the power injected into the batteries is consumed by the load (Fig. 10A). When the discharge current is high (Idech=6A) between $10 \mathrm{~h}$ and $11 \mathrm{~h}$, VBAT and SoC decrease, since the power injected in the batteries does not compensate this consumed by the load.

- All the results obtained in this section show the good control of energy transferred during charge cycle, discharge cycle and charge/discharge cycle of batteries depending on the use. This demonstrates the validation of the simulation results and therefore of the proposed charge/discharge technique of the PV system conceived (Fig. 5).
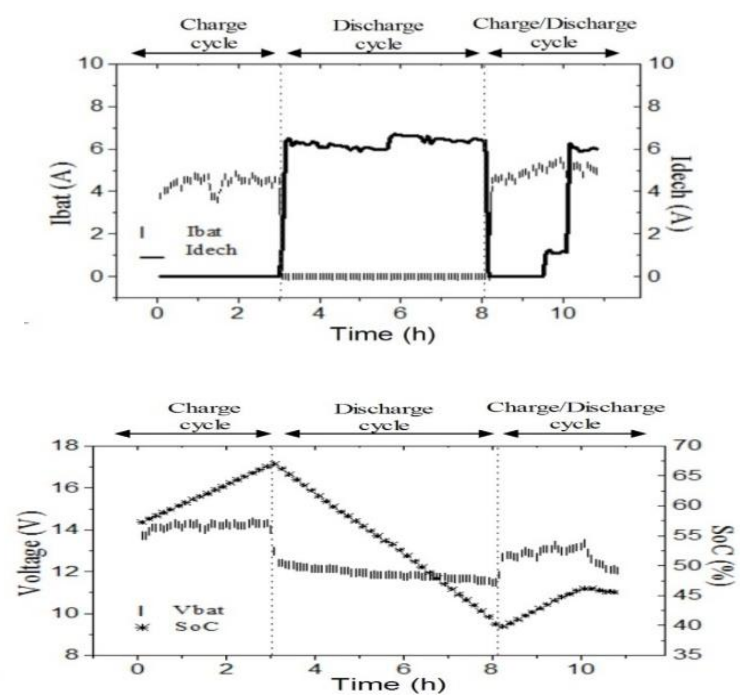

Fig .10 The evolution of electrical characteristics of the batteries during a charge cycle. (A): Variation of VBAT and SoC. (B): Variation of IBAT and Idech 


\section{Optimal functioning of the PV system}

Concerning the optimal functioning of the PV system, where HILL CLIMBING improved algorithm is adopted (Fig. 4), the PWM signal that ensures the MPPT control, the search time to converge to the MPP and generated by the MSS and the electrical quantities (voltage, current and power) of PV panels and batteries during their charging/discharging process are extracted. From these results, the efficiency of the panels ( $\square$ panel), DC/DC converter ( $\square \mathrm{cnv}$ ) and global PV system ( $\square$ sysPV) are inferred. Taking into consideration the same graphs, the results of optimal simulation obtained in Pspice under the same conditions are reported [12]. All the results obtained (Fig. 11 to Fig. 16) show that:

- There is a very good agreement between the results of optimal simulation and the experimental ones.

- The duty cycle of the PWM signal generated by the MSS system by using HILL CLIMBING improved algorithm, to ensure the MPPT control (Fig. 11) is practically the optimum one. The rapidity of the system convergence to the MPP is tested by experimentation and comparison of this algorithm to the classical presented in reference [11]. The results (Fig. 12) showed that the search time ratio of the classical (Tcla) and improved algorithms (Timp) clearly depends on the irradiance and the position of the functioning point of the PV panel. The improved algorithm is much faster and the search time can reach $50 \%$ compared to the search time using the classical algorithm.

- The electrical quantities (voltages, currents and powers) of the PV panels (Fig. 13) are practically the optimum ones, throughout all the batteries charging process. This shows that the MPPT controller has performed its role. It optimizes the functioning of the panels without divergence.

- The efficiency of the PV panels is around $9.5 \%$ (Fig. 14). This shows, firstly, a good agreement between the type of PV panels used and their ageing and, secondly, low losses of the power supplied by the PV panels while the installation is working.

- During the first phase (Boost) of charging (0-38h), the DC/DC converter efficiency (Fig. 15) is the efficiency of the battery charging since all the power supplied by the batteries is absorbed. This efficiency, which is about $85 \%$, is very satisfactory and close to optimum.

- During the second and third phases of charging (38h - 55h), the efficiency while injecting into the grid increases and that of the batteries charge decreases (Fig. 15). This is so due to the decrease (increase) of the power absorbed (injected) by the batteries (grid).

- The global efficiency of the PV system during the charging and the injection into the grid (Fig. 16) is around $8.5 \%$. It is very satisfactory and considered as the best percentage according to the literature [13-14].

- During the functioning of the PV system, the batteries are charged in three phases determined by the algorithm of Fig. 4 that take into consideration the parameters set (Thresholds charges MPPT control, power circuits ...). The different results obtained show the reliability of the system designed, in addition, the efficiencies presented in Fig. 14 to Fig. 16 guarantee the performance of the various blocks of the PV system: DC / DC converters, power system, etc.

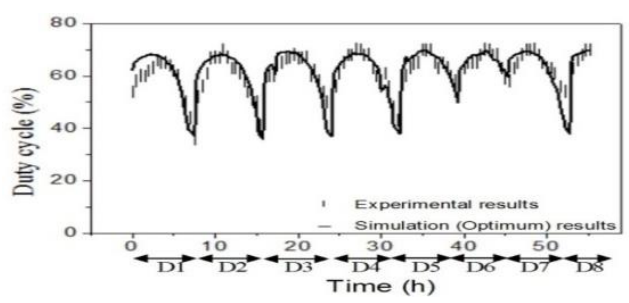

Fig .11 The evolution of the duty cycle as a function of time.

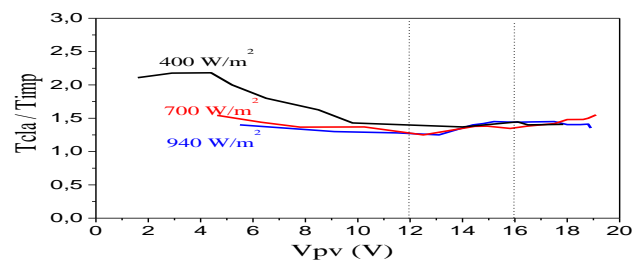

Fig .12 Relationship between the search times of the MPP in the presence of the classic and improved algorithms in function of the Vpv and the irradiance 

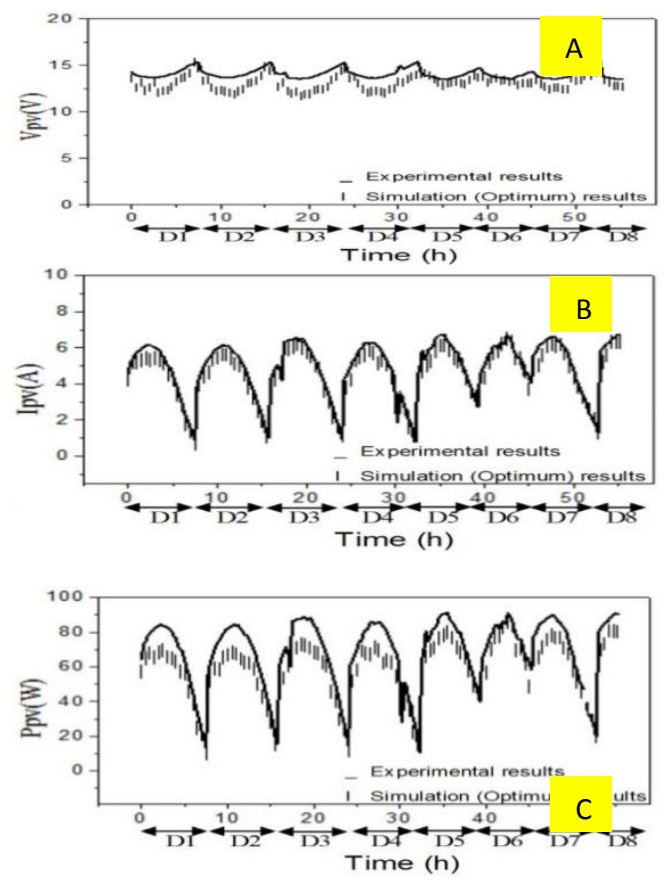

Fig .13 The evolution of: (A): experimental and simulated (Optimum) results of Vpv voltage (B): experimental and simulated (Optimum) results of Ipv current. (C): experimental and simulated (Optimum) results of Ppv power

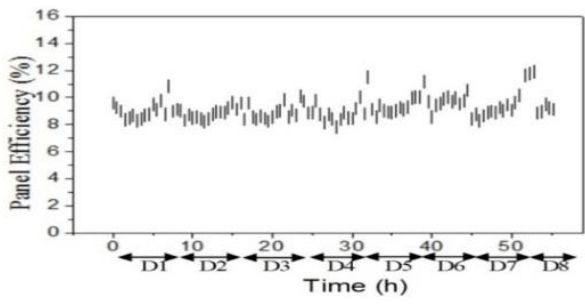

Fig .14 Efficiency of the PV Panels.

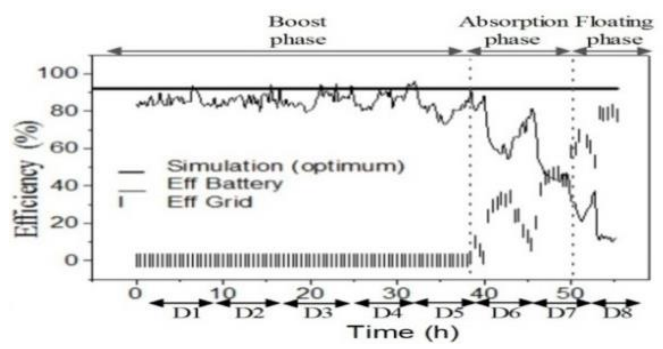

Fig .15 Efficiency of DC/DC converter (experimental and simulation (optimum) results) during the charging and the injection into the grid.

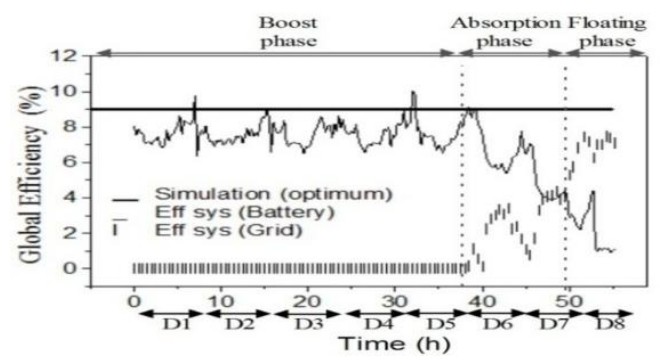

Fig .16 The global efficiency of PV system (experimental and simulation (optimum) results) during the charging and the injection into the grid.

\section{CONCLUSION}

In this paper, the designed photovoltaic system is studied, which is equipped with a management and digital control system. The experimentation shows that the PV system works under optimal conditions and the search time of the MPP is significantly improved by $50 \%$ compared to the search time by using the classical algorithm. The PV system becomes fast and accurate. Also, the batteries are fully charged in three phases with a good estimation of the state of charge (around 5\% of improvement as compared to the traditional method). During this operation, the control circuit of energetic flow allows to efficiently manage the energy supplied by the PV panels and to inject the surplus into the grid when the batteries are charged and even during the charging phases while the regulation is in order to minimize losses and for better use of energy. All this is accompanied by a good performance of the converter and the global PV system (85\% and $8.5 \%)$.

The global results obtained in this work demonstrate the reliability and optimization of the PV system. Firstly, the digital card of the management and supervision system is configured according to the technical characteristics of this equipment ( $P V$ panels, batteries ...) and, secondly, he uses algorithms which are adaptable to all situations such as changes in illumination, loads and temperature. 


\section{ACKNOWLEDGEMENTS}

This work is supported by:

- Belgian Development Agency CTB (Project MIP/012/010), Morocco.

- United Nations Development Programme UNDP Art Gold Morocco, ENV 2008200.

- Cooperation Moroccan-Belgian "Institutional University Commission", IUC, Oujda, 20082012 (Water and Environment Activity / Sub-Activity Renewable Energy).

\section{REFERENCES}

[1] Eftichios Koutroulis, and Frede Blaabjerg, "Design optimization of transformerless gridConnected PV inverters including reliability," IEEE TRANSACTIONS ON POWER ELECTRONICS, Vol. 28, no. 1, Jan 2013, p. 325.

[2] Rodolfo Dufo-López , Juan M. Lujano-Rojas, José L. Bernal-Agustín, "Comparison of different lead-acid battery lifetime prediction models for use in simulation of stand-alone photovoltaic systems," Applied Energy, 115 (2014), pp. 242253.

[3] Hongbin Wu, Shuzhao Wang1, Bo Zhao2 and Chengzhi Zhu2, "Energy management and control strategy of a grid-connected PV/battery system," International Transactions on Electrical Energy Systems Article first published online: 18 April 2014, DOI: 10.1002/etep.1926.

[4] Mehdi Dali a, Jamel Belhadj, Xavier Roboam, "Hybrid solar-wind system with battery storage operating in grid-connected and standalone mode: Control and energy management experimental investigation, Energy, 35 (2010), pp. 2587-2595.

[5] Chang Yoon Chun a, Jongbok Baek a, Gab-Su Seo a, B.H. Cho a, *, Jonghoon Kim b, II Kwon Chang c, Sangwoo Lee c, "Current sensor-less state-of-charge estimation algorithm for lithiumion batteries utilizing filtered terminal voltage," Journal of Power Sources, 273 (2015), pp. 255263.

[6] Saeed Sepasi a, ${ }^{*}$, Reza Ghorbani a, Bor Yann
Liaw b, "A novel on-board state-of-charge estimation method for aged Li-ion batteries based on model adaptive Extended Kalman filter," Journal of Power Sources, 245 (2014), pp. 337-344.

[7] K. Hirech, M. Melhaoui, F. Yaden, E. Baghaz, K. Kassmi, "Design and realization of an autonomous system equipped with a regulator of charge/discharge and digital MPPT command," The Mediterranean Green Energy Forum 2013, MGEF-13.

[8] M. Melhaoui, E. Baghaz, K. Hirech, F. Yaden, Khalil Kassmi, "Contribution to the improvement of the MPPT control functioning of photovoltaic systems," International Review of Electrical Engineering (IREE), [S.I.], v. 9, n. 2, pp. 393400, apr. 2014. ISSN 1827-6679.

[9] Kong-Soon Ng, Yao Feng Huang, Chin-Sien Moo, and Yao-Ching Hsieh, "An enhanced Coulomb counting method for estimating stateof-charge and state-of-health of lead-acid batteries," IEEE Transaction. Published in Telecommunications Energy Conference, 2009. INTELEC 2009. 31st International.

[10] Hongxing Yang, Zhaohong Fang, Wei Zhou, "Battery behavior prediction and battery working states analysis of a hybrid solar-wind power generation system," Renewable Energy 33, 2008, pp. 1413-1423.

[11] Baghaz, M. Melhaoui, F. Yaden, K. Hirech, K. Kassmi. "Design, realization and optimization of the photovoltaic systems equipped with analog and digital MPPT commands," Energy Procedia, 42 (2013).

[12] Web-page : http://www.cadence.com, 2014.

[13] Jung Hun So, Young SeokJung, Gwon Jong Yu, Ju Yeop Choi, Jae Ho Choi, "Performance results and analysis of $3 \mathrm{~kW}$ grid-connected PV systems, Renewable Energy 32, 2007, pp. 1858-1872.

[14] Danny H.W. Li, K.L. Cheung, Tony N.T. Lam, Wilco W.H. Chan, "A study of grid-connected photovoltaic (PV) system in Hong Kong," Applied Energy, 90, 2012, pp. 122-127. Contents lists available at Science Direct Applied Energy. 